\title{
Erratum to: On Different-Dimensional Deployment Problems of Hybrid VANET-Sensor Networks with QoS Considerations
}

\author{
Chun-Cheng Lin ${ }^{1} \cdot$ Peng-Chung Chen ${ }^{1} \cdot$ Li-Wei Chang ${ }^{1}$
}

Published online: 3 March 2016

(C) Springer Science+Business Media New York 2016

\section{Erratum to: Mobile Netw Appl}

DOI 10.1007/s11036-015-0667-3

The original version of this article unfortunately contained mistakes. The changes are enumerated below:

1. "Hsing-Wen Wang" should be deleted from the author group section.

2. "Chun-Cheng Lin" should be the corresponding author of this article.

3. Algorithms 1, 2, and 3 should be presented as:

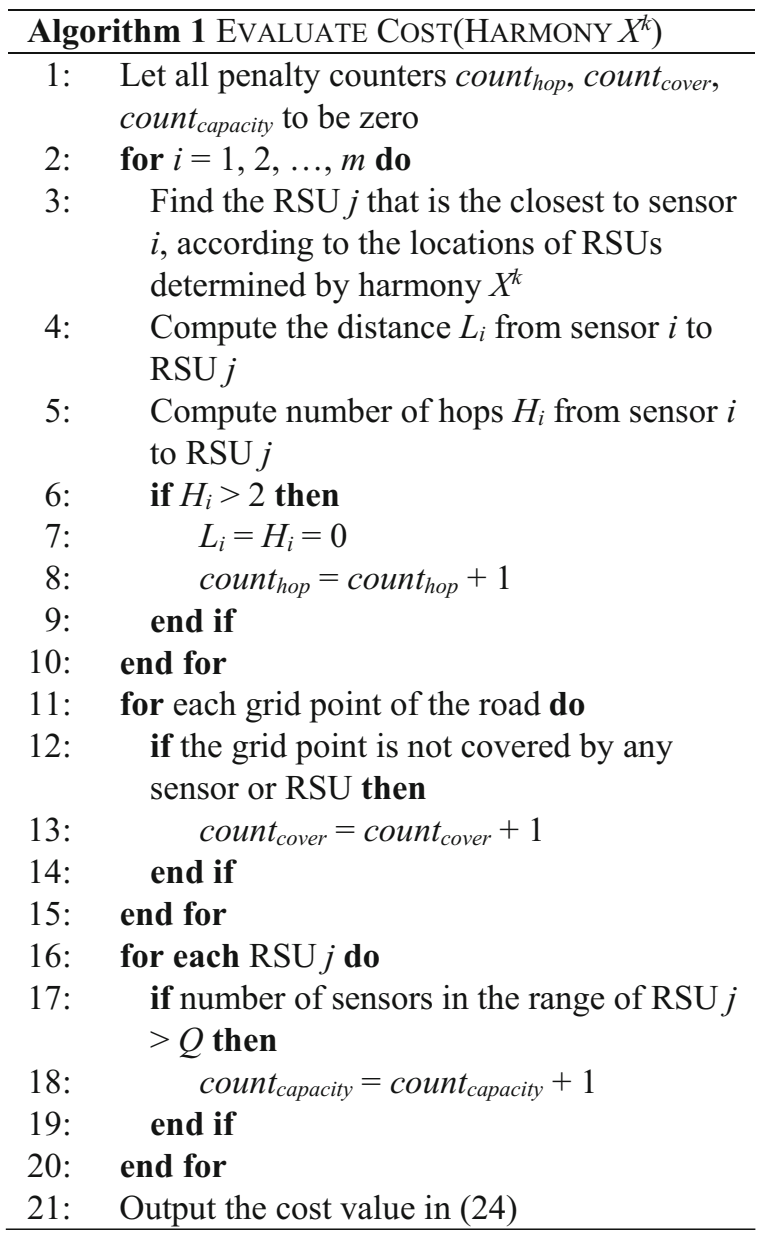



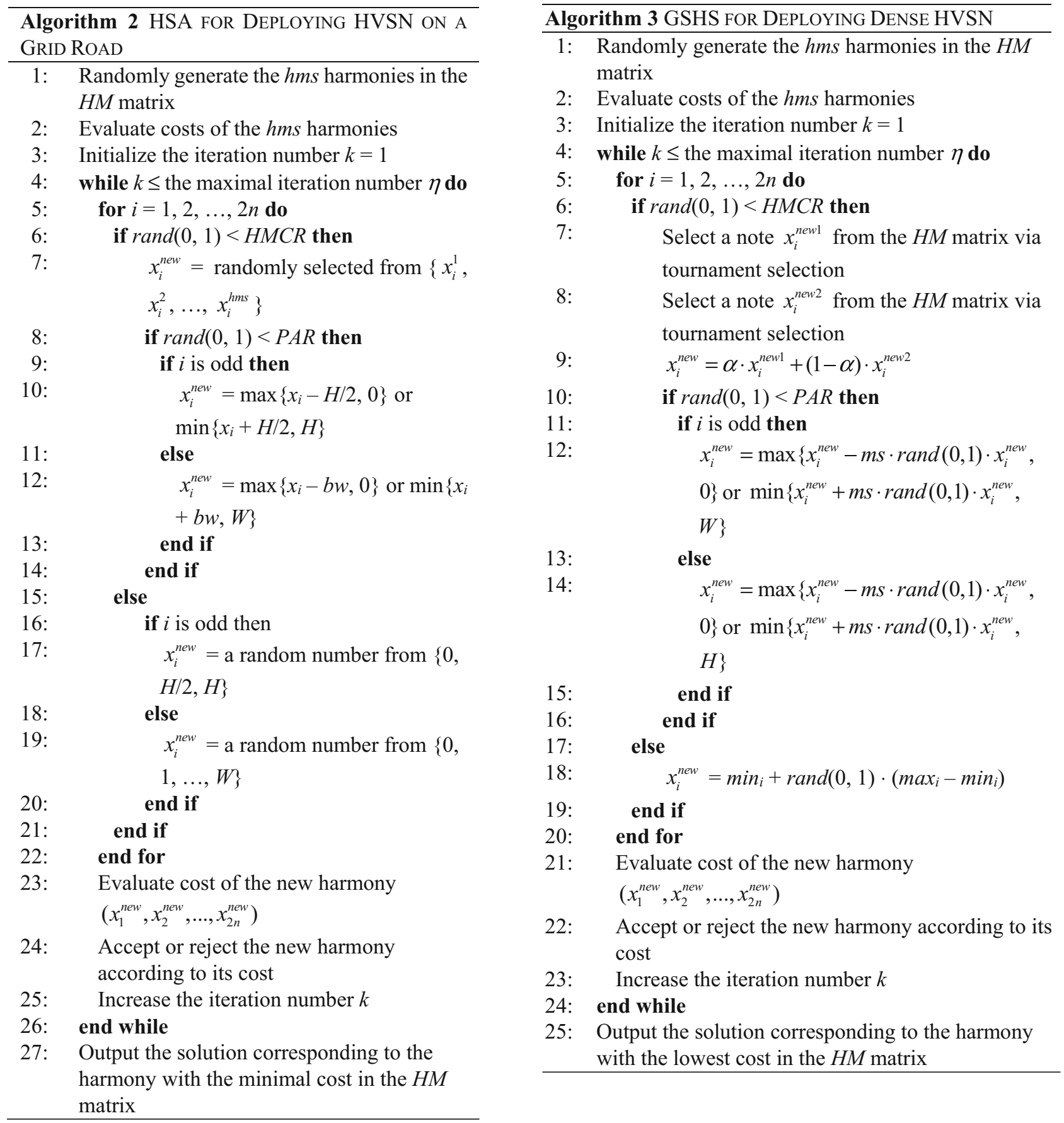
4. Page 7, a square symbol should be added at the end of the following sentences:

Hence, all the three loops in Algorithm 1 can be done in $O(H+m+H \times W+n)=O(H \times W+m+n)$.

Hence, the loop with $\eta$ iterations in Lines 3-26 can be done in $O(\eta \cdot(2 n+H \cdot W+m+n+h m s))=O(\eta \cdot(H \cdot W+m+n+h m s))$.
5. Page 9, Equation 26 should be presented as:

$$
\frac{\lambda \sum_{i=1}^{m} L_{i} /\left(C_{R}+C_{S}\right)+(1-\lambda) \sum_{i=1}^{m} H_{i} / 2}{m-\text { count }_{\text {hop }}}
$$

Note: The original version of this article was corrected based on the above enumerated changes. 